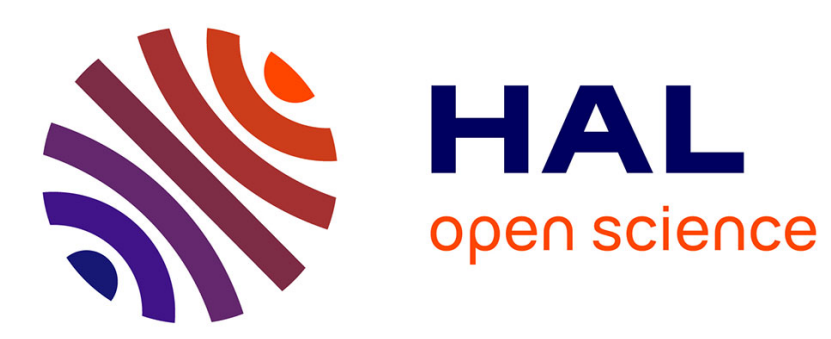

\title{
Oriented Coloring of Triangle-Free Planar Graphs and 2-Outerplanar Graphs
}

\author{
Pascal Ochem, Alexandre Pinlou
}

\section{To cite this version:}

Pascal Ochem, Alexandre Pinlou. Oriented Coloring of Triangle-Free Planar Graphs and 2Outerplanar Graphs. LAGOS'11: VI Latin-American Algorithms, Graphs and Optimization Symposium, Mar 2011, Bariloche, Argentina. pp.123-128, 10.1016/j.endm.2011.05.022 . lirmm-00530543

\section{HAL Id: lirmm-00530543 https://hal-lirmm.ccsd.cnrs.fr/lirmm-00530543}

Submitted on 29 Oct 2010

HAL is a multi-disciplinary open access archive for the deposit and dissemination of scientific research documents, whether they are published or not. The documents may come from teaching and research institutions in France or abroad, or from public or private research centers.
L'archive ouverte pluridisciplinaire HAL, est destinée au dépôt et à la diffusion de documents scientifiques de niveau recherche, publiés ou non, émanant des établissements d'enseignement et de recherche français ou étrangers, des laboratoires publics ou privés. 


\title{
Oriented coloring of triangle-free planar graphs and 2-outerplanar graphs ${ }^{1}$
}

\author{
Pascal Ochem ${ }^{\mathrm{a}}$ Alexandre Pinlou ${ }^{\mathrm{b}, 2}$ \\ ${ }^{a}$ LRI - Univ. Paris 11, CNRS - Bat. 490, 91405 Orsay Cedex, France \\ ${ }^{\mathrm{b}}$ LIRMM - Univ. Montpellier 2, CNRS - 161 rue Ada, 34392 Montpellier Cedex 5, France
}

\begin{abstract}
A graph is planar if it can be embedded on the plane without edge-crossing. A graph is 2-outerplanar if it has a planar embedding such that the subgraph obtained by removing the vertices of the external face is outerplanar (i.e. with all its vertices on the external face). An oriented $k$-coloring of an oriented graph $G$ is a homomorphism from $G$ to an oriented graph $H$ of order $k$. We prove that every oriented triangle-free planar graph has an oriented chromatic number at most 40, that improves the previous known bound of 47 due to Borodin and Ivanova [Borodin, O. V. and Ivanova, A. O., An oriented colouring of planar graphs with girth at least 4, Sib. Electron. Math. Reports, vol. 2, 239-249, 2005]. We also prove that every oriented 2-outerplanar graph has an oriented chromatic number at most 40, that improves the previous known bound of 67 due to Esperet and Ochem [Esperet, L. and Ochem, P. Oriented colouring of 2-outerplanar graphs, Inform. Process. Lett., vol. 101(5), 215-219, 2005].
\end{abstract}

Key words: Oriented coloring; Planar graph; Girth; 2-outerplanar graph; Discharging procedure.

Email addresses: Pascal.Ochemelri.fr (Pascal Ochem), Alexandre.Pinlou@lirmm.fr (Alexandre Pinlou).

URLs: http://www.lri.fr/ ochem (Pascal Ochem), http://www.lirmm.fr/ pinlou (Alexandre Pinlou).

1 This research is partially supported by the ANR GRATOS, under contract ANR-09JCJC-0041-01.

2 Second affiliation: Département de Mathématiques et Informatique Appliqués, Université Paul-Valéry, Montpellier 3, Route de Mende, 34199 Montpellier Cedex 5, France 


\section{Introduction}

Oriented graphs are directed graphs without loops nor opposite arcs. For an oriented graph $G$, we denote by $V(G)$ its set of vertices and by $A(G)$ its set of arcs. For two adjacent vertices $u$ and $v$, we denote by $\overrightarrow{u v}$ the arc from $u$ to $v$ or simply $u \sim v$ whenever its orientation is not relevant (therefore, $u \sim v=\overrightarrow{u v}$ or $u \sim v=\overrightarrow{v u}$ ). The number of vertices of $G$ is the order of $G$.

An oriented k-coloring of an oriented graph $G$ is a mapping $\varphi$ from $V(G)$ to a set of $k$ colors such that (1) $\varphi(u) \neq \varphi(v)$ whenever $\overrightarrow{u v}$ is an arc in $G$, and (2) $\varphi(u) \neq \varphi(x)$ whenever $\overrightarrow{u v}$ and $\overrightarrow{w x}$ are two arcs in $G$ with $\varphi(v)=\varphi(w)$. In other words, an oriented $k$-coloring of $G$ is a partition of the vertices of $G$ into $k$ stable sets $S_{1}, S_{2}, \ldots, S_{k}$ such that all the arcs between any pair of stable sets $S_{i}$ and $S_{j}$ have the same direction (either from $S_{i}$ to $S_{j}$, or from $S_{j}$ to $S_{i}$ ). The oriented chromatic number of an oriented graph, denoted by $\chi_{o}(G)$, is defined as the smallest $k$ such that $G$ admits an oriented $k$-coloring.

Let $G$ and $H$ be two oriented graphs. A homomorphism from $G$ to $H$ is a mapping $\varphi: V(G) \rightarrow V(H)$ that preserves the arcs: $\overrightarrow{\varphi(x) \varphi(y)} \in A(H)$ whenever $\overrightarrow{x y} \in A(G)$.

An oriented $k$-coloring of $G$ can be equivalently defined as a homomorphism from $G$ to $H$, where $H$ is an oriented graph of order $k$. The existence of such a homomorphism from $G$ to $H$ is denoted by $G \rightarrow H$. The vertices of $H$ are called colors, and we say that $G$ is $H$-colorable. The oriented chromatic number of $G$ can then be defined as the smallest order of an oriented graph $H$ such that $G \rightarrow H$. Links between colorings and homomorphisms are presented in more details in the monograph [7] by Hell and Nešetřil.

The notion of oriented coloring introduced by Courcelle [5] has been studied by several authors in the last decade and the problem of bounding the oriented chromatic number has been investigated for various graph classes: outerplanar graphs (with given girth) [13,15], 2-outerplanar graphs [6], planar graphs (with given girth) $[1-4,10,12,14]$, graphs with bounded maximum average degree [3,4], graphs with bounded degree [8], graphs with bounded treewidth $[11,15,16]$, and graph subdivisions [18].

A graph is planar if it can be embedded on the plane without edge-crossing. The girth of a graph is the length of a shortest cycle.

Theorem 1 gives the current best known bounds on oriented chromatic number of planar graphs.

Theorem 1 [1-4,12] Let $G$ be a planar graph.

(1) If $G$ has girth at least 12, then $\chi_{o}(G) \leq 5$ [3] (this bound is tight). 
(2) If $G$ has girth at least 7, then $\chi_{o}(G) \leq 7$ [1].

(3) If $G$ has girth at least 6, then $\chi_{o}(G) \leq 11$ [4].

(4) If $G$ has girth at least 5, then $\chi_{o}(G) \leq 16$ [12].

(5) If $G$ has girth at least 4 , then $\chi_{o}(G) \leq 47$ [2].

(6) If $G$ has no girth restriction, then $\chi_{o}(G) \leq 80$ [14].

A graph is 2-outerplarnar if it has a planar embedding such that the subgraph obtained by removing the vertices of the external face is outerplanar (i.e. with all its vertices on the external face).

In 2007, Esperet and Ochem [6] studied the structural properties of 2-outerplanar graphs. By means of these properties, they proved the following:

Theorem 2 [6] Let $G$ be a 2-outerplanar graph. Then $\chi_{o}(G) \leq 67$.

As mentioned above, if a graph $G$ admits a homomorphism to an oriented graph $H$ of order $k$, then $G$ has an oriented chromatic number $k$. Hence, a way to get bounds on the oriented chromatic number of a graph family $\mathcal{F}$ is to find a universal target graph $H$ such that, for every graph $G \in \mathcal{F}$, we have $G \rightarrow H$. Such a result can be obtained if the target graph $H$ has "interesting" structural properties that can be used to prove the existence of the homomorphism; thus an important part of the task is to construct such a target graph. In this paper, we first describe the construction of the graph $T_{40}$ in Section 2, an oriented graph on 40 vertices which has very useful properties for oriented coloring of planar graphs.

These structural properties of $T_{40}$ allow us to prove that every oriented trianglefree planar graph admits a homomorphism to $T_{40}$; this gives the following theorem, which improves Theorem 1(5).

Theorem 3 Let $G$ be a triangle-free planar graph. Then $\chi_{o}(G) \leq 40$.

We also show that every oriented 2-outerplanar graph admits a homomorphism to $T_{40}$; this allows us to improves Theorem 2 .

Theorem 4 Let $G$ be a 2-outerplanar graph. Then $\chi_{o}(G) \leq 40$.

In the remainder, we use the following notions. The set of vertices (resp. arcs, faces) of a graph $G$ is denoted by $V(G)$ (resp. $A(G), F(G)$ ). For a vertex $v$ of a graph $G$, we denote by $d_{G}^{-}(v)$ its indegree, by $d_{G}^{+}(v)$ its outdegree, and by $d_{G}(v)$ its degree (subscripts are omitted when the considered graph is clearly identified from the context). We denote by $N_{G}^{+}(v)$ the set of outgoing neighbors of $v$, by $N_{G}^{-}(v)$ the set of incoming neighbors of $v$ and by $N_{G}(v)=N_{G}^{+}(v) \cup N_{G}^{-}(v)$ the set of neighbors of $v$. A vertex of degree $k$ (resp. at least $k$, at most $k$ ) is called a $k$-vertex (resp. ${ }^{\geq} k$-vertex,

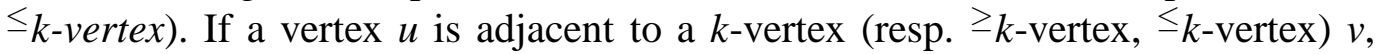
then $v$ is a $k$-neighbor (resp. ${ }^{\geq}$-neighbor, ${ }_{k}$-neighbor) of $u$. A path of length $k$ (i.e. formed by $k$ edges) is called a $k$-path. For a face $f$ of a graph $G$, its length is denoted 
by $d_{G}(f)$ (subscripts are omitted when the considered graph is clearly identified from the context). If $d_{G}(f)=k$ (resp. $\left.d_{G}(f) \leq k, d_{G}(f) \geq k\right)$, then $f$ is called a $k$-face (resp. $\leq k$-face, $\geq k$-face). If two graphs $G$ and $H$ are isomorphic, we denote it by $G \cong H$. Given a planar graph $G$ with its embedding in the plane and a vertex $v$ of $G$, we say that a subset $\left\{u_{1}, u_{2}, \ldots, u_{k}\right\}$ of neighbors of $v$ are consecutive if $u_{1}, u_{2}, \ldots, u_{k}$ appear around $v$ consecutively (clockwise or counterclockwise) in $G$.

The paper is organised as follows. The next section is devoted to the target graph $T_{40}$ and some of its properties. We prove Theorem 3 in Section 3 and Theorem 4 in Section 4.

\section{The Tromp graph $T_{40}$}

In this section, we describe the construction of the target graph $T_{40}$ used to prove Theorems 3 and 4 and give some useful properties.

Tromp's construction was proposed by Tromp [17]. Let $G$ be an oriented graph and $G^{\prime}$ be an isomorphic copy of $G$. The Tromp graph $\operatorname{Tr}(G)$ has $2|V(G)|+2$ vertices and is defined as follows:

- $V(\operatorname{Tr}(G))=V(G) \cup V\left(G^{\prime}\right) \cup\left\{\infty, \infty^{\prime}\right\}$

- $\forall u \in V(G): \overrightarrow{u \infty}, \overrightarrow{\infty u^{\prime}}, \overrightarrow{u^{\prime} \infty^{\prime}}, \overrightarrow{\infty^{\prime} u} \in A(\operatorname{Tr}(G))$

- $\forall u, v \in V(G), \overrightarrow{u v} \in A(G): \overrightarrow{u v}, \overrightarrow{u^{\prime} v^{\prime}}, \overrightarrow{v u^{\prime}}, \overrightarrow{v^{\prime} u} \in A(\operatorname{Tr}(G))$

Figure 1 illustrates the construction of $\operatorname{Tr}(G)$. We can observe that, for every $u \in$ $V(G) \cup\{\infty\}$, there is no arc between $u$ and $u^{\prime}$. Such pairs of vertices will be called twin vertices, and we denote by $t(u)$ the twin vertex of $u$. Remark that $t(t(u))=u$. This notion can be extended to sets in a standard way: for a given $W \subseteq V(G)$, $W=\left\{v_{1}, v_{2}, \ldots, v_{k}\right\}$, then $t(W)=\left\{t\left(v_{1}\right), t\left(v_{2}\right), \ldots, t\left(v_{k}\right)\right\}$.

By construction, the graph $\operatorname{Tr}(G)$ satisfies the following property:

$$
\forall u \in \operatorname{Tr}(G): N^{+}(u)=N^{-}(t(u)) \text { and } N^{-}(u)=N^{+}(t(u))
$$

In the remainder, we focus on the specific graph family obtained via the Tromp's construction applied to Paley tournaments. For a prime power $p \equiv 3(\bmod 4)$, the Paley tournament $Q R_{p}$ is defined as the oriented graph whose vertices are the integers modulo $p$ and such that $\overrightarrow{u v}$ is an arc if and only if $v-u$ is a nonzero quadratic residue of $p$. For instance, the Paley tournament $Q R_{19}$ has vertex set $V\left(Q R_{19}\right)=\{0,1, \ldots, 18\}$ and $\overrightarrow{u v} \in A\left(Q R_{19}\right)$ whenever $v-u \equiv r(\bmod 19)$ for $r \in\{1,4,5,6,7,9,11,16,17\}$. Note that the bounds of Theorems 1(2) and 1(3), have been obtained by proving that all the graphs of the considered classes admit a homomorphism to the Paley tournaments $Q R_{7}$ and $Q R_{11}$, respectively. Moreover, 


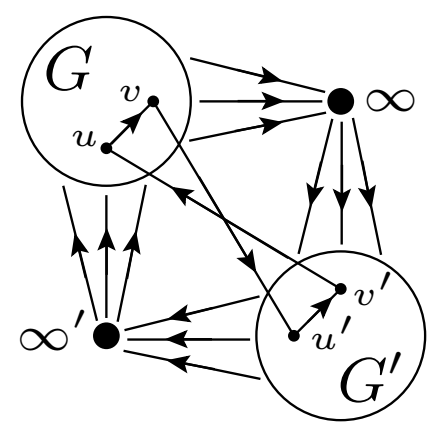

Fig. 1. The Tromp graph $\operatorname{Tr}(G)$.

the bound of Theorem 1(4) has been obtained by proving that all the graphs of the considered class admit a homomorphism to the Tromp graph $\operatorname{Tr}\left(Q R_{7}\right)$.

Let $T_{40}=\operatorname{Tr}\left(Q R_{19}\right)$ be the Tromp graph on 40 vertices obtained from $Q R_{19}$. In the remainder of this paper, the vertex set of $T_{40}$ is $V\left(T_{40}\right)=\left\{0,1, \ldots, 18, \infty, 0^{\prime}, 1^{\prime}, \ldots, 18^{\prime}, \infty^{\prime}\right\}$ where $\{0,1, \ldots, 18\}$ is the vertex set of the first copy of $Q R_{19}$ and $\left\{0^{\prime}, 1^{\prime}, \ldots, 18^{\prime}\right\}$ is the vertex set of the second copy of $Q R_{19}$; thus, for every $u \in\{0,1, \ldots, 18, \infty\}$, we have $t(u)=u^{\prime}$. In addition, for every $u \in V\left(T_{40}\right)$, we have by construction $\left|N_{T_{40}}^{+}(u)\right|=\left|N_{T_{40}}^{-}(u)\right|=19$. The graph $T_{40}$ has remarkable symmetry and some useful properties given below.

Proposition 5 [9] For any $Q R_{p}$, the graph $\operatorname{Tr}\left(Q R_{p}\right)$ is such that:

$$
\forall u \in V\left(\operatorname{Tr}\left(Q R_{p}\right)\right): N^{+}(u) \cong Q R_{p} \text { and } N^{-}(u) \cong Q R_{p}
$$

Proposition 6 [9] For any $Q R_{p}$, if $\left\{a_{1}, a_{2}, a_{3}\right\}$ and $\left\{b_{1}, b_{2}, b_{3}\right\}$ span triangles $t_{1}$ and $t_{2}$ respectively in $\operatorname{Tr}\left(Q R_{p}\right)$ and the map $\psi$ taking $a_{i}$ to $b_{i}(1 \leq i \leq 3)$ is an isomorphism $t_{1} \rightarrow t_{2}$, then $\psi$ can be extended to an automorphism of $\operatorname{Tr}\left(Q R_{p}\right)$.

It is then clear that $\operatorname{Tr}\left(Q R_{p}\right)$ is vertex-transitive and arc-transitive.

For an oriented graph $G$ and a vertex $v$, pushing $v$ means reversing the orientation of every arc incident to $v$.

Proposition 7 (Push Property) Let $G$ be an oriented graph such that $G \rightarrow \operatorname{Tr}\left(Q R_{p}\right)$. Then, for any vertex $v$ of $G$, the graph $G^{\prime}$ obtained from $G$ by pushing $v$ admits a homomorphism to $\operatorname{Tr}\left(Q R_{p}\right)$.

Proof. Let $\varphi$ be a $\operatorname{Tr}\left(Q R_{p}\right)$-coloring of $G$. For every $w \in V\left(\operatorname{Tr}\left(Q R_{p}\right)\right)$, we have $N_{\operatorname{Tr}\left(Q R_{p}\right)}^{+}(w)=N_{\operatorname{Tr}\left(Q R_{p}\right)}^{-}(t(w))$ and $N_{\operatorname{Tr}\left(Q R_{p}\right)}^{-}(w)=N_{\operatorname{Tr}\left(Q R_{p}\right)}^{+}(t(w))$. Therefore, the mapping $\varphi^{\prime}: V\left(G^{\prime}\right) \rightarrow V\left(\operatorname{Tr}\left(Q R_{p}\right)\right)$ defined by $\varphi^{\prime}(u)=\varphi(u)$ for all $u \in V\left(G^{\prime}\right) \backslash\{v\}$ and $\varphi^{\prime}(v)=t(\varphi(v))$ is clearly a $\operatorname{Tr}\left(Q R_{p}\right)$-coloring of $G^{\prime}$.

An orientation $n$-vector is a sequence $\alpha=\left(\alpha_{1}, \alpha_{2}, \ldots, \alpha_{n}\right) \in\{0,1\}^{n}$ of $n$ elements. 
Let $S=\left(v_{1}, v_{2}, \ldots, v_{n}\right)$ be a sequence of $n$ (not necessarily distinct) vertices of $T_{40}$. The vertex $u$ is said to be an $\alpha$-successor of $S$ if for any $i, 1 \leq i \leq n$, we have $\overrightarrow{u v_{i}} \in A\left(T_{40}\right)$ whenever $\alpha_{i}=1$ and $\overrightarrow{v_{i} u} \in A\left(T_{40}\right)$ otherwise. For instance, the vertex $3^{\prime}$ of $T_{40}$ is a $(1,1,0,1,1,0)$-successor of $\left(1,2,6^{\prime}, 1, \infty^{\prime}, 2^{\prime}\right)$ since the arcs $\overrightarrow{3^{\prime} 1}, \overrightarrow{3^{\prime} 2}$, $\overrightarrow{6^{\prime} 3^{\prime}}, \overrightarrow{3^{\prime} \infty^{\prime}}$, and $\overrightarrow{2^{\prime} 3^{\prime}}$ belong to $A\left(T_{40}\right)$.

If, for a sequence $S=\left(v_{1}, v_{2}, \ldots, v_{n}\right)$ of $n$ vertices of $T_{40}$ and an orientation $n$ vector $\alpha=\left(\alpha_{1}, \alpha_{2}, \ldots, \alpha_{n}\right)$, there exist $i \neq j$ such that $v_{i}=v_{j}$ and $\alpha_{i} \neq \alpha_{j}$, then there does not exist any $\alpha$-successor of $S$; indeed, $T_{40}$ does not contain opposite arcs. In addition, if there exist $i \neq j$ such that $v_{i}=t\left(v_{j}\right)$ and $\alpha_{i}=\alpha_{j}$, then there does not exist any $\alpha$-successor of $S$; indeed, for any pair of vertices $x$ and $y$ of $T_{40}$ with $x=t(y)$, we have $N_{T_{40}}^{+}(x) \cap N_{T_{40}}^{+}(y)=\emptyset$ and $N_{T_{40}}^{-}(x) \cap N_{T_{40}}^{-}(y)=\emptyset$. A sequence $S=\left(v_{1}, v_{2}, \ldots, v_{n}\right)$ of $n$ vertices of $T_{40}$ is said to be compatible with an orientation $n$-vector $\alpha=\left(\alpha_{1}, \alpha_{2}, \ldots, \alpha_{n}\right)$ if and only if for any $i \neq j$, we have $\alpha_{i} \neq \alpha_{j}$ whenever $v_{i}=t\left(v_{j}\right)$, and $\alpha_{i}=\alpha_{j}$ whenever $v_{i}=v_{j}$. Note that if the $n$ vertices of $S$ induce an $n$ clique subgraph of $T_{40}$ (i.e. $v_{1}, v_{2}, \ldots, v_{n}$ are pairwise distinct and induce a complete graph), then $S$ is compatible with any orientation $n$-vector since a vertex $u$ and its twin $t(u)$ cannot belong together to the same clique.

In the remainder, we say that $T_{40}$ has Property $P_{n, k}$ if, for every sequence $S$ of $n$ vertices of $T_{40}$ that form an $n$-clique and any orientation $n$-vector $\alpha$ which is compatible with $S$, there exist $k \alpha$-successors of $S$.

Proposition 8 If, for a fixed $\alpha=\left(\alpha_{1}, \alpha_{2}, \ldots, \alpha_{n}\right)$, every $n$-clique $S$ of $T_{40}$ admits $k$ $\alpha$-successors, then there exist $k \alpha^{\prime}$-successors of $S$ for every $\alpha^{\prime}=\left(\alpha_{1}^{\prime}, \alpha_{2}^{\prime}, \ldots, \alpha_{n}^{\prime}\right)$.

Proof. Assume that every $n$-clique admit $k \alpha$-successors. Let $S=\left(u_{1}, u_{2}, \ldots, u_{n}\right)$ be a $n$-clique of $T_{40}$ and $\alpha^{\prime}=\left(\alpha_{1}^{\prime}, \alpha_{2}^{\prime}, \ldots, \alpha_{n}^{\prime}\right)$ be an orientation $n$-vector. Then let $S^{\prime}=\left(v_{1}, v_{2}, \ldots, v_{n}\right)$ defined such that $v_{i}=u_{i}$ if $\alpha_{i}^{\prime}=\alpha_{i}$ and $v_{i}=t\left(u_{i}\right)$ otherwise. Due to the structure of $T_{40}$ (i.e. if $x \sim y$ belongs to $A\left(T_{40}\right)$, then $t(x) \sim y, x \sim t(y)$ and $t(x) \sim t(y)$ belongs to $\left.A\left(T_{40}\right)\right), S^{\prime}$ is an $n$-clique of $T_{40}$. By hypothesis, $S^{\prime}$ admits $k \alpha$-successors $w_{1}, w_{2}, \ldots, w_{k}$. Since $\overrightarrow{y t(x)} \in A\left(T_{40}\right)$ if $\overrightarrow{x y} \in A\left(T_{40}\right)$, we clearly have that $w_{i}$ is an $\alpha^{\prime}$-successor of $S$ for every $i$.

Proposition 9 The graph $T_{40}$ has Properties $P_{1,19}, P_{2,9}, P_{3,4}$, and $P_{4,1}$.

Proof. By Proposition 5, we have $\left|N^{+}(u)\right|=\left|N^{-}(u)\right|=19$ for every vertex $u$ of $T_{40}$; therefore $T_{40}$ has Property $P_{1,19}$.

It is obvious that $Q R_{19}$ has properties $P_{1,9}$ (for every vertex $u$ of $Q R_{19}$, we have $\left|N^{+}(u)\right|=\left|N^{-}(u)\right|=9$ ). Borodin et al. [4] proved that $Q R_{19}$ has properties $P_{2,4}$ and $P_{3,1}$. We will show in the remainder of this proof that if $Q R_{19}$ has properties $P_{n-1, k}$, then $T_{40}$ have property $P_{n, k}$, that will complete the proof. 
Suppose that $Q R_{19}$ has property $P_{n-1, k}$ and let $\alpha=\left(\alpha_{1}, \alpha_{2}, \ldots, \alpha_{n}\right)$ be a given orientation $n$-vector. Let $S=\left(u_{1}, u_{2}, \ldots, u_{n-1}, w\right)$ be a induced $n$-clique of $T_{40}$. If $\alpha_{n}=0$, we define $S^{\prime}=\left(v_{1}, v_{2}, \ldots, v_{n-1}, w\right)$ such that $v_{i}=u_{i}$ if $\overrightarrow{u_{i} w}$ and $v_{i}=t\left(u_{i}\right)$ if $\overrightarrow{w u_{i}}$. Hence, $S^{\prime}$ is an $n$-clique of $T_{40}$ such that $\bigcup_{i} v_{i} \subseteq N^{-}(w)$. By Proposition $5, N^{-}(w)=$ $K_{19} \cong Q R_{19}$, and therefore the $(n-1)$-clique $S^{\prime \prime}=\left(v_{1}, v_{2}, \ldots, v_{n-1}\right)$ belongs to $K_{19}$. Then by Property $P_{n-1, k}$ of $Q R_{19}$, there exist $k\left(\alpha_{1}^{\prime}, \alpha_{2}^{\prime}, \ldots, \alpha_{n-1}^{\prime}\right)$-successors $x_{1}, x_{2}, \ldots, x_{k}$ of $S^{\prime \prime}$ in $K_{19}$, with $\alpha_{i}^{\prime}=\alpha_{i}$ (resp. $\left.\alpha_{i}^{\prime}=1-\alpha_{i}\right)$ if $u_{i}=v_{i}$ (resp. $u_{i}=t\left(v_{i}\right)$ ).

The $x_{i}$ 's are clearly in-neighbors of $w$ and hence, they are $\left(\alpha_{1}^{\prime}, \alpha_{2}^{\prime}, \ldots, \alpha_{n-1}^{\prime}, \alpha_{n}\right)$ successors $S^{\prime}$, and thus there exist $k \alpha$-successors of $S$. Proportion 8 allows us to conclude.

The case $\alpha_{n}=1$ would be treated similarly: We would have chosen $S^{\prime}=\left(v_{1}, v_{2}, \ldots, v_{n-1}, w\right)$ is such a way that $\bigcup_{i} v_{i} \subseteq N^{+}(w)$.

\section{Proof of Theorem 3}

In this section, we prove Theorem 3, that is, every oriented triangle-free planar graph $G$ admits a homomorphism to $T_{40}$.

Recall that Borodin et al. [2] proved that every oriented triangle-free planar graph $G$ admits a homomorphism to $Q R_{47}$. This proof was only published in Russian. Our proof is highly inspired from the above-mentioned paper. Indeed, our list of forbidden configurations is designed to fit with Borodin's discharging procedure up to a slight modification in Rule (R3).

Let us define the partial order $\preceq$. Let $n_{3}(G)$ be the number of $\geq 3$-vertices in $G$. For any two graphs $G_{1}$ and $G_{2}$, we have $G_{1} \prec G_{2}$ if and only if at least one of the following conditions hold:

- $\left|V\left(G_{1}\right)\right|<\left|V\left(G_{2}\right)\right|$ and $n_{3}\left(G_{1}\right) \leq n_{3}\left(G_{2}\right)$.

- $n_{3}\left(G_{1}\right)<n_{3}\left(G_{2}\right)$.

Note that the partial order $\preceq$ is well-defined and is a partial linear extension of the induced subgraph poset.

Let $H$ be a hypothetical minimal counterexample to Theorem 3 according to $\prec$. We first prove that $H$ does not contain a set of ten configurations listed in Lemma 10. Then, using a discharging procedure, we show that an oriented triangle-free planar graph contains at least one of the ten configurations of Lemma 10, contradicting the fact that $H$ is a triangle-free planar graph. 


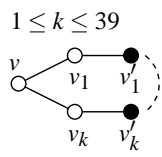

(a) $\mathrm{C} 2$

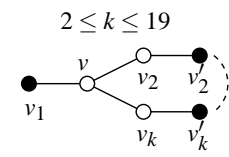

(b) $\mathrm{C} 3$

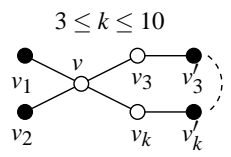

(c) $\mathrm{C} 4$

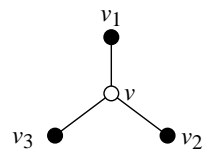

(d) $\mathrm{C} 5$

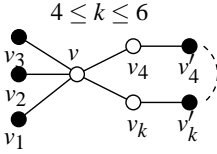

(e) $\mathrm{C6}$

Fig. 2. Configurations $C 2-C 6$.

\section{$3.1 \quad$ Structural properties of $H$}

In the following, $H$ is a triangle-free planar graph given with its embedding in the plane. A weak 7-vertex $u$ in $H$ is a 7-vertex adjacent to four 2-vertices $v_{1}, \ldots, v_{4}$ and three $\geq 3$-vertices $w_{1}, w_{2}, w_{3}$ in such a way that the sequence of neighbors of $v$ appear as $v_{1}, w_{1}, v_{2}, w_{2}, v_{3}, w_{3}, v_{4}$ (clockwise or counterclockwise).

Lemma 10 The graph $H$ does not contain the following configurations:

(C1) $a \leq 1$-vertex;

(C2) a k-vertex adjacent to $k 2$-vertices for $2 \leq k \leq 39$;

(C3) a k-vertex adjacent to $(k-1) 2$-vertices for $2 \leq k \leq 19$;

(C4) a $k$-vertex adjacent to $(k-2) 2$-vertices for $3 \leq k \leq 10$;

(C5) a 3-vertex;

(C6) a k-vertex adjacent to $(k-3) 2$-vertices for $3 \leq k \leq 6$;

(C7) two vertices $u$ and $v$ linked by three distinct 2-paths whose the internal vertex of two of them is a 2-vertex;

(C8) two vertices $u$ and $v$ linked by two distinct 2-paths whose the internal vertex them is a 2-vertex;

(C9) a 4-face wxyz such that $x$ is 2-vertex, $w$ and $y$ are weak 7-vertices, and $z$ is a $k$-vertex adjacent to $(k-3) 2$-vertices for $3 \leq k \leq 8$;

(C10) a 4-face wxyz such that $x$ is 2-vertex, $w$ and $y$ are weak 7-vertices, and $z$ is a $k$-vertex adjacent to $(k-4) 2$-vertices for $4 \leq k \leq 7$;

The drawing conventions for a configuration $C$ contained in a graph $G$ are the following. If $u$ and $v$ are two vertices of $C$, then they are adjacent in $G$ if and only if they are adjacent in $C$. Moreover, the neighbors of a white vertex in $G$ are exactly its neighbors in $C$, whereas a black vertex may have neighbors outside of $C$. Two or more black vertices in $C$ may coincide in a single vertex in $G$, provided they do not share a common white neighbor. Finally, an edge will represent an arc with any of its two possible orientations. Configurations $(C 2)-(C 10)$ are depicted in Figures 2 and 3.

Let $G$ be an oriented graph, $v$ be a $k$-vertex with $N(v)=\left\{v_{1}, v_{2}, \ldots, v_{k}\right\}$ and $\alpha$ be an orientation $k$-vector such that $\alpha_{i}=0$ whenever $\overrightarrow{v_{i} v} \in A(G)$ and $\alpha_{i}=1$ otherwise. Let $\varphi$ be a $T_{40}$-coloring of $G \backslash\{v\}$ and $S=\left(\varphi\left(v_{1}\right), \varphi\left(v_{2}\right), \ldots, \varphi\left(v_{k}\right)\right)$. Recall that a necessary condition to have $\alpha$-successors of $S$ is that $\alpha$ must be compatible with $S$, that is for any pair of vertices $v_{i}$ and $v_{j}, \varphi\left(v_{i}\right) \neq \varphi\left(v_{j}\right)$ whenever $\alpha_{i} \neq \alpha_{j}$ and 
$\varphi\left(v_{i}\right) \neq t\left(\varphi\left(v_{j}\right)\right)$ whenever $\alpha_{i}=\alpha_{j}$. Hence, every vertex $v_{j}$ forbids one color for each vertex $v_{i}, i \in[1, k], i \neq j$. We define $f_{v_{i}}^{\varphi}\left(v_{j}\right)$ to be the forbidden color for $v_{i}$ by $\varphi\left(v_{j}\right)$ (i.e. $f_{v_{i}}^{\varphi}\left(v_{j}\right)=\varphi\left(v_{j}\right)$ whenever $\alpha_{i} \neq \alpha_{j}$ and $f_{v_{i}}^{\varphi}\left(v_{j}\right)=t\left(\varphi\left(v_{j}\right)\right)$ whenever $\left.\alpha_{i}=\alpha_{j}\right)$. Therefore, $\alpha$ is compatible with $S$ if and only if we have $\varphi\left(v_{i}\right) \neq f_{v_{i}}^{\varphi}\left(v_{j}\right)$ for every pair $i, j, 1 \leq i<j \leq k$. Note that if $\varphi\left(v_{i}\right) \neq f_{v_{i}}^{\varphi}\left(v_{j}\right)$, then we necessarily have $\varphi\left(v_{j}\right) \neq f_{v_{j}}^{\varphi}\left(v_{i}\right)$.

For each configuration, we suppose that $H$ contains it and we consider a trianglefree reduction $H^{\prime}$ such that $H^{\prime} \prec H$; therefore, by minimality of $H, H^{\prime}$ admits a $T_{40}$-coloring $\varphi$. We will then show that we can choose $\varphi$ so that it can be extended to $H$ by Proposition 9, contradicting the fact that $H$ is a counterexample.

In the remainder, if $H$ contains a configuration, then $H^{*}$ will denote the graph obtained from $H$ be removing all the white vertices from this configuration.

Proof of Configuration $(C 1)$. Trivial.

Proof of Configuration $(C 2)$. Suppose that $H$ contains the configuration depicted in Figure 2(a) and let $\varphi$ be a $T_{40}$-coloring of $H^{*}$. Let $F=\left\{f_{v}^{\varphi}\left(v_{1}^{\prime}\right), \ldots, f_{v}^{\varphi}\left(v_{k}^{\prime}\right)\right\}$ be the set of forbidden colors for $v$. Any $T_{40}$-coloring of $H^{*}$ can be extended to $H$ since $|F| \leq 39$.

Proof of Configuration $(C 3)$. Suppose that $H$ contains the configuration depicted in Figure 2(b) and let $\varphi$ be a $T_{40}$-coloring of $H^{*}$. Let $F=\left\{f_{v}^{\varphi}\left(v_{1}^{\prime}\right), \ldots, f_{v}^{\varphi}\left(v_{k}^{\prime}\right)\right\}$ be the set of forbidden colors for $v$. By Property $P_{1,19}, \varphi$ can be extended to $H$ since $|F| \leq 18$.

Proof of Configuration $(C 4)$. Suppose that $H$ contains the configuration depicted in Figure 2(c) and let $\varphi$ be a $T_{40}$-coloring of $H^{\prime}=H \backslash\left\{v_{3}, \ldots, v_{k}\right\}$. Then, we clearly have $\varphi\left(v_{1}\right) \neq f_{v_{1}}^{\varphi}\left(v_{2}\right)$ since $v$ is colored in $H^{\prime}$. Therefore, by Property $P_{2,9}$, there exist an $T_{40}$-coloring $\varphi^{\prime}$ of $H^{\prime}$ so that $\varphi^{\prime}(v) \notin\left\{f_{v}^{\varphi^{\prime}}\left(v_{3}^{\prime}\right), \ldots, f_{v}^{\varphi^{\prime}}\left(v_{k}^{\prime}\right)\right\}$. The coloring $\varphi^{\prime}$ can be extended to $H$.

Proof of Configuration $(C 5)$. Suppose that $H$ contains the configuration depicted in Figure 2(d). Let $H^{\prime}$ be the graph obtained from $H^{*}$ by adding, for every $1 \leq i<$ $j \leq 3$, a 2-path joining $v_{i}$ to $v_{j}$ with the same orientation as the path $\left[v_{i}, v, v_{j}\right]$ in $H$. Since Configurations $(C 1)-(C 4)$ are forbidden, $d_{H}\left(v_{i}\right) \geq 3$ for $1 \leq i \leq 3$; we thus have $H^{\prime} \prec H$ since $n_{3}\left(H^{\prime}\right)=n_{3}(H)-1$, and $H^{\prime}$ is clearly triangle-free. Any $T_{40}$-coloring $\varphi$ of $H^{\prime}$ induces a coloring of $H^{*}$ such that $\varphi\left(v_{i}\right) \neq f_{v_{i}}^{\varphi}\left(v_{j}\right)$ for any $i, j$, $1 \leq i<j \leq 3$. Then Property $P_{3,4}$ allows us to extend $\varphi$ to $H$. 


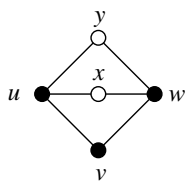

(a) $\mathrm{C} 7$

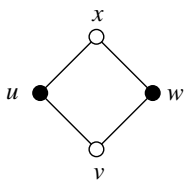

(b) $\mathrm{C} 8$

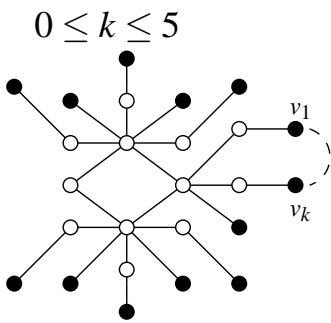

(c) $\mathrm{C} 9$

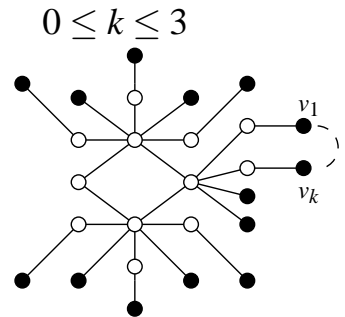

(d) $\mathrm{C} 10$

Fig. 3. Configurations $C 7-C 10$.

Proof of Configuration (C6). Suppose that $H$ contains the configuration depicted in Figure 2(e). Let $\varphi$ be a $T_{40}$-coloring of $H^{\prime}=H \backslash\left\{v_{4}, \ldots, v_{k}\right\}$. Then, we clearly have $\varphi\left(v_{i}\right) \neq f_{v_{i}}^{\varphi}\left(v_{j}\right)$, for all $1 \leq i \leq j \leq 3$, since $v$ is colored in $H^{\prime}$. Therefore, by Property $P_{3,4}$, there exists a $T_{40}$-coloring $\varphi^{\prime}$ of $H^{\prime}$ such that $\varphi^{\prime}(v) \notin\left\{f_{v}^{\varphi^{\prime}}\left(v_{4}^{\prime}\right), \ldots, f_{v}^{\varphi^{\prime}}\left(v_{k}^{\prime}\right)\right\}$.

Proof of Configuration $(C 7)$. Suppose that $H$ contains the configuration depicted in Figure 3(a). Let $H^{\prime}$ be the graph obtained from $H^{*}$ by adding a 2-path $u v^{\prime} w$ between $u$ and $w$ such that $u v^{\prime} w$ is directed if and only if $u v w$ is not directed. We have that $H^{\prime} \prec H$ since $\left|V\left(H^{\prime}\right)\right|=|V(H)|-1$ and $n_{3}\left(H^{\prime}\right)=n_{3}(H)$. Due to the orientations of the 2-paths $u v^{\prime} w$ and $u v w$, any $T_{40}$-coloring $\varphi$ of $H^{\prime}$ ensures that $\varphi(u) \neq \varphi(w)$ and $\varphi(u) \neq t(\varphi(w))$. The coloring $\varphi$ can be extended to $H$.

Proof of Configuration (C8). Suppose that $H$ contains the configuration depicted in Figure 3(b). Let $H^{\prime}$ be the graph obtained from $H^{*}$ by adding an edge between $u$ and $w$. We have that $H^{\prime} \prec H$ since $\left|V\left(H^{\prime}\right)\right|=|V(H)|-2 n_{3}\left(H^{\prime}\right)=n_{3}(H)$. Since Configuration $(C 7)$ is forbidden, the vertices $u$ and $w$ are at distance at least 3 in $H^{*}$ and $H^{\prime}$ is therefore triangle-free. Any $T_{40}$-coloring $\varphi$ of $H^{\prime}$ ensures that $\varphi(u) \neq \varphi(w)$ and $\varphi(u) \neq t(\varphi(w))$. The coloring $\varphi$ can be extended to $H$.

Proof of Configurations $(C 9)$ and $(C 10)$. To prove that these two configurations are forbidden in a minimal counterexample to Theorem 3 , a computer check is needed. Indeed, Properties $P_{1,19}, P_{2,9}, P_{3,4}$ and $P_{4,1}$ are not sufficient.

A computer check allows us to show that for any compatible color assignment on the black vertices (i.e. any two black vertices at distance 2 in the configuration get compatible colors) and any orientation of the arcs, the white vertices can be colored. Therefore, that shows that $H$ does not contain any of these two configurations. 


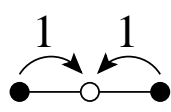

(a) R1

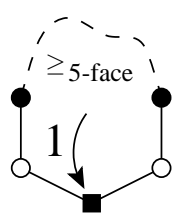

(b) R2

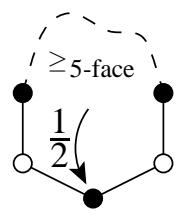

(c) R2

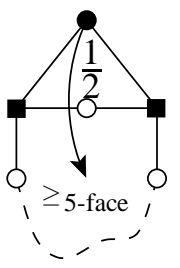

(d) R3

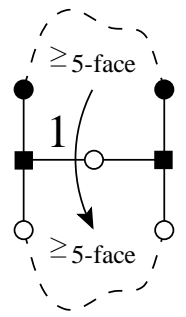

(e) R3

Fig. 4. Discharging rules

\subsection{Discharging procedure}

To complete the proof of Theorem 3, we use a discharging procedure. We define the weight function $\omega$ by $\omega(x)=d(x)-4$ for every $x \in V(H) \cup F(H)$. Since $H$ is a planar graph, we have by Euler formula $(|V(H)|-|A(H)|+|F(H)|=2)$ :

$$
\sum_{v \in V(H)} \omega(v)+\sum_{f \in F(H)} \omega(f)=\sum_{v \in V(H)}(d(v)-4)+\sum_{f \in F(H)}(d(f)-4)=-2<0 .
$$

In what follows, we will define discharging rules (R1), (R2), and (R3) and redistribute weights accordingly. Once the discharging is finished, a new weight function $\omega^{*}$ is produced. However, the total sum of weights is fixed by the discharging rules. Nevertheless, we can show that $\omega^{*}(v) \geq 0$ for every $x \in V(H) \cup F(H)$. This leads to the following obvious contradiction:

$$
0 \leq \sum_{v \in V(H)} \omega^{*}(v)+\sum_{f \in F(H)} \omega^{*}(f)=\sum_{v \in V(H)} \omega(v)+\sum_{f \in F(H)} \omega(f)<0 .
$$

Therefore, no such counterexample $H$ exists.

The discharging rules are defined as follows:

(R1) Each $\geq 4$-vertex gives 1 to its 2-neighbors.

(R2) Each $\geq 5$-face ...axb... such that $a$ and $b$ are 2-vertices gives 1 (resp. $\frac{1}{2}$ ) to $x$ if $x$ is a weak 7-vertices (resp. is not a weak 7-vertex).

(R3) Each $\geq 5$-face $f=\ldots a w x y b \ldots$, such that $a, b, x$ are 2 -vertices and $w, y$ are weak 7 -vertices, either receives $\frac{1}{2}$ from the vertex $z$ if $w x y z$ is a 4 -face, or receives 1 from the $\geq 5$-face $f^{\prime}=\ldots$ cwxyd $\ldots$ if $c, d$ are $\geq 4$-vertices.

The discharging rules are illustrated in Figure 4: white disks (resp. black disks, black squares) are 2 -vertices (resp. ${ }^{\geq}$-vertices, weak 7 -vertices). 


\subsubsection{For all vertices $v, \omega^{*}(v) \geq 0$}

In the following, $d_{\geq 4}(v)$ denotes the number of neighbors of $v$ with degree at least 4 . In the same way, $d_{2}(v)$ denotes the number of neighbors of $v$ with degree exactly 2 . Then it is clear that, for every vertex $v$ of $H$, we have $d(v)=d_{\geq 4}(v)+d_{2}(v)$ since $H$ contains neither vertices of degree at most 1 by $(C 1)$, nor 3 -vertices by $(C 5)$.

Let $v$ be a $k$-vertex of $H$. Therefore, $k=d_{\geq 4}(v)+d_{2}(v)$. Recall that the initial charge of $v$ is $\omega(v)=k-4$.

- if $d_{\geq 4}(v)=0$, then $d_{2}(v)=k \geq 40$ by $(C 2)$. By (R1), $v$ gives $k \times 1$. By $(C 8), v$ is incident to $k \geq 5$-faces, and therefore $v$ receives $k \times \frac{1}{2}$ by (R2). Hence, $\omega^{*}(v)=$ $\omega(v)-k+\frac{k}{2} \geq 16$.

- if $d_{\geq 4}(v)=1$, then $d_{2}(v)=k-1 \geq 19$ by (C3). By (R1), $v$ gives $(k-1) \times 1$. By $(C 8), v$ is incident to $(k-2) \geq 5$-faces whose each gives $\frac{1}{2}$ to $v$ by (R2). Moreover, $v$ is adjacent to at most one weak 7-vertex and therefore (R3) does not apply. Hence, $\omega^{*}(v)=\omega(v)-(k-1)+\frac{k-2}{2} \geq 6$.

- if $d_{\geq 4}(v)=2$, then $d_{2}(v)=k-2 \geq 9$ by $(C 4)$. By (R1), $v$ gives $(k-2) \times 1$. By $(C 8), v$ is incident to $(k-4) \geq 5$-faces whose each gives $\frac{1}{2}$ to $v$ by (R2). Moreover, by (R3), $v$ gives at most $\frac{1}{2}$ since $v$ is adjacent to at most two weak 7 -vertices. Hence, $\omega^{*}(v)=\omega(v)-(k-2)+\frac{k-4}{2}-\frac{1}{2} \geq 1$.

- if $d_{\geq 4}(v)=3$, then $d_{2}(v)=k-3 \geq 4$ by $(C 5)$ and (C6). In each case, by (R1), $v$ gives $(k-3) \times 1$.

- Suppose that the three $\geq 4$-neighbors are consecutive. By $(C 8), v$ is incident to $(k-4) \geq 5$-faces whose each gives $\frac{1}{2}$ to $v$ by (R2). Moreover, by (R3), $v$ gives at most $2 \times \frac{1}{2}$ if and only if $d_{2}(v) \geq 6$, that implies $k \geq 9$ by $(C 9)$. Hence, if $k \leq 8, \omega^{*}(v)=\omega(v)-(k-3)+\frac{k-4}{2} \geq \frac{1}{2}$; if $k \geq 9, \omega^{*}(v)=\omega(v)-(k-3)+$ $\frac{k-4}{2}-2 \cdot \frac{1}{2} \geq \frac{1}{2}$.

- Suppose that two $\geq 4$-neighbors are consecutive. By $(C 8), v$ is incident to $(k-$ 5 ) $\geq 5$-faces whose each gives $\frac{1}{2}$ to $v$ by (R2). Moreover, by (R3), $v$ gives at most $\frac{1}{2}$ if and only if $d_{2}(v) \geq 6$, that implies $k \geq 9$ by (C9). Hence, if $k \leq 8$, $\omega^{*}(v)=\omega(v)-(k-3)+\frac{k-5}{2} \geq 0$; if $k \geq 9, \omega^{*}(v)=\omega(v)-(k-3)+\frac{k-5}{2}-\frac{1}{2} \geq$ $\frac{1}{2}$.

- Suppose that none of the $\geq 4$-neighbors are consecutive. By $(C 8), v$ is incident to $(k-6) \geq 5$-faces whose each gives $\frac{1}{2}$ to $v$ by (R2) if $d(v) \geq 8$ or gives 1 to $v$ by (R2) if $d(v)=7$ (i.e. $v$ is a weak 7-vertex). Moreover, (R3) does not apply. Hence, if $d(v)=7, \omega^{*}(v)=\omega(v)-(k-3)+1=0$; if $d(v) \geq 8, \omega^{*}(v)=$ $\omega(v)-(k-3)+\frac{k-6}{2} \geq 0$.

- If $d_{\geq 4}(v)=4$, then $d_{2}(v)=k-4$. By $(C 1), v$ gives $(k-4) \times 1$.

Suppose that (R3) does not apply. Then, $\omega^{*}(v) \geq \omega(v)-(k-4)=0$. Suppose now that (R3) applies: it applies at most twice (otherwise, it would imply that a weak 7-vertex had three consecutive 2-neighbors). Moreover, by $(C 10)$, we have $d_{2}(v) \geq 4$, that implies $k \geq 8$.

- Suppose first that (R3) applies only once; then $v$ gives $\frac{1}{2}$ to the corresponding 
4-face. Moreover, by (R2), $v$ receives $\frac{k-7}{2}$. Hence, $\omega^{*}(v)=\omega(v)-(k-4)+$ $\frac{k-7}{2}-\frac{1}{2} \geq 0$.

- Suppose now that (R3) applies twice; then $v$ gives $2 \times \frac{1}{2}$ to the corresponding 4-faces. Moreover, by (R2), $v$ receives $\frac{k-6}{2}$. Hence, $\omega^{*}(v)=\omega(v)-(k-4)+$ $\frac{k-6}{2}-2 \times \frac{1}{2} \geq 0$.

- Suppose finally that $d_{\geq 4}(v) \geq 5$. By $(C 1)$, $v$ gives $\left(k-d_{\geq 4}(v)\right) \times 1$. Moreover, by (R3), $v$ gives at most $\frac{1}{2} \times\left\lfloor\frac{d_{\geq 4}(v)}{2}\right\rfloor$. Hence, $\omega^{*}(v) \geq \omega(v)-\left(k-d_{\geq 4}(v)\right)-\frac{1}{2} \times$ $\left\lfloor\frac{d_{>4}(v)}{2}\right\rfloor \geq 0$.

Thus, for every $v \in V(H)$, we have $\omega^{*}(v) \geq 0$ once the discharging is finished.

\subsubsection{For all faces $f, \omega^{*}(f) \geq 0$}

Let $f$ be a $k$-face of $H$. Since $H$ is triangle-free, then $k \geq 4$. Recall that the initial charge of $f$ is $\omega(f)=k-4$.

- If $k=4$, then no rule applies. Hence, $\omega^{*}(f)=\omega(f)=0$

- If $k=5$, then $f$ is incident to at most two 2-vertices by (C3). If $f$ has no incident 2-vertices, then $\omega^{*}(v) \geq \omega(f)=1$.

If $f$ is incident to one 2-vertex, then only (R3) may apply and hence $\omega^{*}(f) \geq$ $\omega(f)-1=0$.

If $f$ is adjacent to two 2-vertices $x$ and $z$, either the common neighbor $y$ of $x$ and $z$ is weak 7-vertex either it is not. By (R2), $f$ gives at most 1 , and hence $\omega^{*}(v) \geq \omega(f)-1=0$.

- If $k=6$, then $f$ is incident to at most three 2-vertices by (C3). If $f$ has no incident 2-vertices, then $\omega^{*}(v) \geq \omega(f)=2$.

If $f$ is incident to one 2-vertex, then only (R3) may apply and hence $\omega^{*}(f) \geq$ $\omega(f)-1=1$.

Suppose that $f$ is incident to two 2-vertex $x$ and $z$. If $x$ and $z$ has a common neighbor $y$, by (R2), $f$ gives at most 1 , and hence $\omega^{*}(v) \geq \omega(f)-1=0$. If $x$ and $z$ has no common neighbor, then only (R3)may apply at most twice. Hence, $\omega^{*}(v) \geq \omega(f)-2 \times 1=0$

Finally, suppose that $f$ is adjacent to three 2-vertices.

- If $f$ is incident to at most one weak 7-vertex, then $f$ gives at most $1 \times 1+2 \times$ $\frac{1}{2}=2$ by (R2). Hence, $\omega^{*}(v) \geq \omega(f)-2=0$.

- If $f$ is incident to two weak 7-vertices, then $f$ gives $2 \times 1+1 \times \frac{1}{2}=\frac{5}{2}$ by (R2). Moreover, $f$ receives at least $\frac{1}{2}$ by (R3). Hence, $\omega^{*}(v) \geq \omega(f)-\frac{5}{2}+\frac{1}{2}=0$.

- If $f$ is incident to three weak 7-vertices, then $f$ gives $3 \times 1$ by (R2). Moreover, $f$ receives at least $3 \times \frac{1}{2}$ by $(\mathrm{R} 3)$. Hence, $\omega^{*}(v) \geq \omega(f)-3+3 \times \frac{1}{2}=\frac{1}{2}$.

- Suppose finally that $k \geq 7$, and assume that (R2) applies $n$ times and (R3) applies $m$ times. It is clear that $f$ gives weights by (R2) to at most $\left\lfloor\frac{k}{2}\right\rfloor$ vertices: hence, $n \leq\left\lfloor\frac{k}{2}\right\rfloor$. Moreover, we can easily check that we have $2 n+3 m \leq k$. With these 


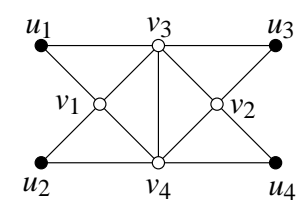

Fig. 5. Unavoidable configuration in a 2-outerplanar graph containing neither a $\leq 3$-vertex, nor two adjacent 4 -vertices

constraints, we have $n+m=\frac{n+2 n+3 m}{3} \leq \frac{\left\lfloor\frac{k}{2}\right\rfloor+k}{3}$, which implies that $n+m \leq$ $k-4$ when $k \geq 7$. Hence, $\omega^{*}(v) \geq \omega(f)-n-m \geq 0$.

Thus, for every $f \in F(H)$, we have $\omega^{*}(v) \geq 0$ once the discharging is finished, that complete the proof.

\section{Proof of Theorem 4}

In this section, we prove Theorem 4, that is every oriented 2-outerplanar graph $G$ admits a homomorphism to $T_{40}$.

Esperet and Ochem [6] proved the following structural theorem for 2-outerplanar graphs.

Theorem 11 [6] Let $G$ be a 2-outerplanar graph. Then $G$ contains either $a \leq 3$ vertex, or two adjacent 4-vertices, or the configuration depicted in Figure 5.

Moreover, the class of 2-outerplanar graphs is a minor-closed graph class.

To prove Theorem 4, we will consider a minimal counterexample and prove that it cannot contain one of the configuration described in Theorem 11, a contradiction.

Let $H$ be a hypothetical minimal counterexample (with respect to the minor order) to Theorem 4.

- It is trivial to show that $H$ does not contain a 1-vertex.

- Suppose that $H$ contains a 2-vertex $v$ adjacent to $u_{1}$ and $u_{2}$. Let $H^{\prime}$ be the graph obtained from $H$ by contracting the arc $u_{1} v$. By minimality of $H$, the graph $H^{\prime}$ admits a $T_{40}$-coloring $\varphi$, and since $u_{1}$ and $u_{2}$ are adjacent in $H^{\prime}, \varphi\left(u_{1}\right) \neq \varphi\left(u_{2}\right)$ and $\varphi\left(u_{1}\right) \neq t\left(\varphi\left(u_{2}\right)\right)$. By $P_{2,9}, \varphi$ can be extended to $H$, a contradiction.

- Suppose that $H$ contains a 3-vertex $v$ adjacent to $u_{1}, u_{2}$, and $u_{3}$. If $v$ is a sink, let $H^{\prime}=H$; otherwise, let $H^{\prime}$ be the graph obtained from $H$ by pushing $u_{1}$ and/or $u_{2}$ and/or $u_{3}$ in such a way that $v$ becomes a sink in $H^{\prime}$ (i.e. $\overrightarrow{u_{1} v}, \overrightarrow{u_{2} v}, \overrightarrow{u_{3} v} \in A\left(H^{\prime}\right)$ ). By the Push Property (Proposition 7), the graph $H^{\prime}$ is clearly a minimal counterexample to Theorem 4 since $H^{\prime}$ is $T_{40}$-colorable if and only of $H$ does.

Suppose first that the subgraph induced by $u_{1}, u_{2}$, and $u_{3}$ in $H^{\prime}$ contains a 


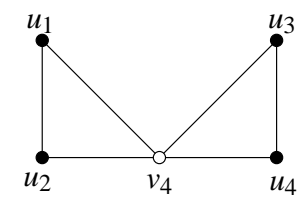

Fig. 6. Reduction of the configuration depicted in Figure 5

sink, say $u_{1}$. Then, let $H^{\prime \prime}$ be the graph obtained from $H^{\prime}$ by contracting $\overrightarrow{u_{1} v}$. By minimality of $H^{\prime}$, the graph $H^{\prime \prime}$ admits a $T_{40}$-coloring $\varphi$. Since $\overrightarrow{u_{2} u_{1}}, \overrightarrow{u_{3} u_{1}} \in$ $A\left(H^{\prime \prime}\right)$, then either the three vertices $\varphi\left(u_{1}\right), \varphi\left(u_{2}\right), \varphi\left(u_{3}\right)$ form a 3-clique in $T_{40}$ or they form a 2-clique in $T_{40}$ with $\varphi\left(u_{2}\right)=\varphi\left(u_{3}\right)$ (recall that $N^{+}(u) \cap N^{+}(t(u))=\emptyset$ for every $u$ of $T_{40}$ ). By $P_{3,4}$, the coloring $\varphi$ can be extended to $H^{\prime}$.

Suppose now that the subgraph induced by $u_{1}, u_{2}$, and $u_{3}$ in $H^{\prime}$ does not contain a sink; then, $u_{1}, u_{2}, u_{3}$ form a directed cycle. Let $H^{\prime \prime}=H^{\prime} \backslash\{v\}$. By minimality of $H^{\prime}$, the graph $H^{\prime \prime}$ admits a $T_{40}$-coloring $\varphi$. It is clear that $\varphi\left(u_{1}\right), \varphi\left(u_{2}\right)$ and $\varphi\left(u_{3}\right)$ form a 3-clique in $T_{40}$. By $P_{3,4}$, the coloring $\varphi$ can be extended to $H^{\prime}$.

Therefore, by the Push Property (Proposition 7 ), $H$ admits a $T_{40}$-coloring, a contradiction.

- Suppose that $H$ contains two adjacent 4-vertices $u$ and $v$ and let $H^{\prime}=H \backslash\{\overrightarrow{u v}\}$. Let $u_{1}, u_{2}, u_{3}$ (resp. $v_{1}, v_{2}, v_{3}$ ) denote the three neighbors of $u$ (resp. $v$ ) distinct from $v$ (resp. $u$ ). By minimality of $H, H^{\prime}$ admits a $T_{40}$-coloring $\varphi$. Then, erase the colors of $u$ and $v$. By $P_{3,4}$, we can color $u$ to get $\varphi(u) \notin \bigcup_{i=1,2,3} f_{u}^{\varphi}\left(v_{i}\right)$. Then by $P_{4,1}$, there exist an available color to extend $\varphi$ to $H$, a contradiction.

- Suppose that $H$ contains the configuration depicted in Figure 5. Let $H^{\prime}$ be the graph obtained from $H$ by contracting the arc $u_{1} v_{1}, v_{3} v_{4}$ and $u_{3} v_{2}$ : we get the graph $H^{\prime}$ depicted in Figure 6. By minimality of $H, H^{\prime}$ admits a $T_{40}$-coloring $\varphi$. By $P_{3,4}$, we can choose $\varphi\left(v_{3}\right) \notin\left\{f_{v_{3}}^{\varphi}\left(u_{1}\right), f_{v_{3}}^{\varphi}\left(v_{4}\right), f_{v_{3}}^{\varphi}\left(u_{3}\right)\right\}$. Then, by $P_{4,1}$, we can color $v_{1}$ and $v_{2}$, a contradiction.

Therefore, $H$ does not contain any of the configurations described in Theorem 11, a contradiction that proves Theorem 4 .

\section{References}

[1] O. V Borodin and A. O Ivanova. An oriented 7-colouring of planar graphs with girth at least 7. Sib. Electron. Math. Reports, 2:222-229, 2005.

[2] O. V Borodin and A. O Ivanova. An oriented colouring of planar graphs with girth at least 4. Sib. Electron. Math. Reports, 2:239-249, 2005.

[3] O. V Borodin, A. O Ivanova, and A. V Kostochka. Oriented 5-coloring of sparse plane graphs. J. Applied and Industrial Mathematics, 1(1):9-17, 2007.

[4] O. V Borodin, A. V Kostochka, J. Nešetřil, A. Raspaud, and É Sopena. On the maximum average degree and the oriented chromatic number of a graph. Discrete Math., 206:77-89, 1999. 
[5] B. Courcelle. The monadic second order-logic of graphs VI : on several representations of graphs by relational structures. Discrete Appl. Math., 54:117-149, 1994.

[6] L. Esperet and P. Ochem. Oriented coloring of 2-outerplanar graphs. Inform. Process. Lett., 101(5):215-219, 2007.

[7] P. Hell and J. Nešetřil. Graphs and homomorphisms, volume 28 of Oxford Lecture Series in Mathematics and its Applications. Oxford University Press, 2004.

[8] A. V. Kostochka, É. Sopena, and X. Zhu. Acyclic and oriented chromatic numbers of graphs. J. Graph Theory, 24:331-340, 1997.

[9] T. H Marshall. Homomorphism bounds for oriented planar graphs. J. Graph Theory, 55(3):175-190, 2007.

[10] P. Ochem. Oriented colorings of triangle-free planar graphs. Inform. Process. Lett., 92:71-76, 2004.

[11] P. Ochem and A. Pinlou. Oriented vertex and arc colorings of partial 2-trees. In European Conference on Combinatorics, Graph Theory and Applications (EuroComb '07), volume 29 of Elect. Notes in Discrete Math., pages 195-199, 2007.

[12] A. Pinlou. An oriented coloring of planar graphs with girth at least five. Discrete Math., 309(8):2108-2118, 2009.

[13] A. Pinlou and É Sopena. Oriented vertex and arc colorings of outerplanar graphs. Inform. Process. Lett., 100(3):97-104, 2006.

[14] A. Raspaud and É Sopena. Good and semi-strong colorings of oriented planar graphs. Inform. Process. Lett., 51(4):171-174, 1994.

[15] É Sopena. The chromatic number of oriented graphs. J. Graph Theory, 25:191-205, 1997.

[16] É Sopena. Oriented graph coloring. Discrete Math., 229(1-3):359-369, 2001.

[17] J. Tromp. Unpublished manuscript.

[18] D. R Wood. Acyclic, star and oriented colourings of graph subdivisions. Discrete Math. Theoret. Comput. Sci., 7(1):37-50, 2005. 